\title{
Stresserfahrungen des „modernen“ Lebens führen zu kognitiven Beeinträchtigungen
}

Fragestellung: Wie beeinflusst multimodaler Kurzzeitstress die Gedächtnisleistung und den Beginn beziehungsweise das Fortschreiten einer Alzheimer-Demenz?

Hintergrund: Epidemiologischen Studien zufolge könnten negative Lebenserfahrungen wie chronischer Stress Risikofaktoren für die Entwicklung einer Alzheimer-Demenz sein. Eine Fehlregulation der HPA-Achse sowie erhöhte Kortisolmengen in der Zerebrospinalflüssigkeit wurden bei Alzheimer-Patienten beschrieben (z. B. [1]). Glukokortikoide und CRH (Corticotrophin releasing hormone) steigern zudem die Produktion von AbetaPeptiden, zentralen Molekülen der Alzheimer-Pathogenese [2]. Die Simulation alläglicher Stressbedingungen im Tierversuchsmodell ist eine Herausforderung, da sowohl psychologische, soziale als auch physische Parameter berücksichtigt werden sollten.

Baglietto-Vargas D, Chen Y, Suh $D$ et al. Short-term modern life-like stress exacerbates A $\beta$ pathology and synapse loss in 3xTg-AD mice. J Neurochem 2015; doi: 10.1111/jnc.13195. [Epub ahead of print]
Methodik: Fünf bis sechs Monate alte Alzheimer-ModellMäuse (3xTg-AD) und nicht transgene Wildtyp-Mäuse wurden für fünf Stunden allein, auf engem Raum und unter starkem Schütteln sehr hellem Licht und einem lau- ten Störgeräusch $(<80 \mathrm{~dB})$ ausgesetzt. Acht Stunden später wurde die Hippokampus-abhängige, kognitive Funktion mit dem Novel-Object-Recognition-Verhaltenstest analysiert. Als Maß für die synaptische Plastizität wurden die dendritischen Ausläufer hippokampaler Neurone analysiert, zudem erfolgten biochemische Analysen bezüglich der Abeta-Peptidsynthese.

Ergebnisse: Die Gedächtnisleistung gestresster Wildtyp-Mäuse war im Vergleich zu nicht gestressten Mäusen beeinträchtigt. 3xTg-AD-Mäuse, die bereits eine stark beeinträchtigte Lern- und Gedächtnisleistung aufwiesen, verschlechterten sich durch den Stress nicht weiter. Die synaptische Plastizität war in AlzheimerModell-Mäusen unter Stressbedingungen signifikant geringer als bei unbehandelten Mäusen. Auch zeigten diese Tiere erhöhte Mengen an Abeta-Peptiden, was auf eine Steigerung des synthetisierenden Enzyms (Beta-Sekretase) und somit einer gesteigerten Produktion zurückzuführen war; gleichzeitig wurde ein CRH-Anstieg beobachtet. Abeta abbauende Proteine sowie Komponenten der Tau-vermittelten Pathologie blieben unbeeinflusst.

Schlussfolgerungen: Die Simulation komplexer Stresssituationen induziert in einem Alzheimer-Mausmodell eine verstärkte Abeta-Bildung sowie eine Reduktion synapsenhaltiger dendritischer Dornen und der kognitiven Leistung.

\section{- Kommentar von Kristina Endres, Mainz}

\section{Stressvermeidung als Demenzprävention}

Die Autoren beschreiben ein neues Modell, mit dem komplexe Stresssituationen in Mäusen simuliert werden können. Unter den hier gewählten multimodalen Stressbedingungen konnten die Autoren zeigen, dass in transgenen Tieren die Abeta-Synthese verstärkt und die synaptische Plastizität sowie die Gedächtnisleistung vermindert wurden. Vorhergehende Studien belegen jedoch, dass Variablen wie der zeitliche Abstand des Lernereignisses zur Stresserfahrung, das daraus resultierende Level von Noradrenalin und Glukokortikoiden sowie die emotionale Valenz des Lerninhalts die Richtung des Effekts von Stress auf das Gedächtnis maßgeblich beeinflussen [3]. Es ist daher noch nicht geklärt - wie auch die Autoren selbst diskutieren -, ob sich das frühe wiederkehrende Auftreten komplexer Stresserfahrungen auf die Entwicklung einer Alzheimer-Demenz im Alter auswirkt. Zudem ist die Übertragbarkeit des Stressmodells auf den Menschen fraglich, da hier in relativ drastischer Weise emotionaler mit starkem physischen Stress direkt verknüpft wurde und die Auswirkung von Stress in einem vergleichsweise starken Tiermodell (Überexpression einer mutierten APP- und Tau-Variante) untersucht wurde. Dennoch könnten sich neue Therapieansätze durch die Vermeidung von negativen Stresserfahrungen beziehungsweise durch Stärkung von resilienzvermittelnden Strategien ableiten lassen, wenn sich der Zusammenhang zwischen Abeta-Synthese und Stress beim Menschen bestätigt.
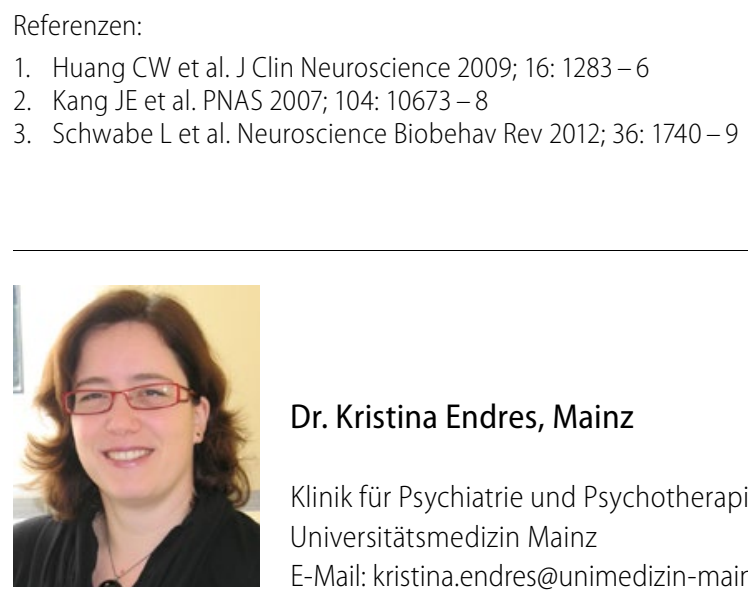

Dr. Kristina Endres, Mainz

Klinik für Psychiatrie und Psychotherapie, Universitätsmedizin Mainz E-Mail: kristina.endres@unimedizin-mainz.de 